\title{
Single Nucleotide Polymorphism in Coding Sequence
}

National Cancer Institute

\section{Source}

National Cancer Institute. Single Nucleotide Polymorphism in Coding Sequence. NCI

Thesaurus. Code C18518.

Single Nucleotide Polymorphisms in Coding Sequences consist of a variation at an appreciable frequency between individuals of a single interbreeding population of a single nucleotide, due to base substitution, at an equivalent location within a protein-coding region of a gene that may or may not cause an amino acid change in the protein product and a detectable phenotypic effect. 